\title{
Anormale Augenstellung als Aussage in der bildenden Kunst Europas
}

\author{
Elfriede Stangler-Zuschrott
}

Eingegangen: 25. Oktober 2017 / Angenommen: 9. Januar 2018 / Online publiziert: 9. Februar 2018

(C) Der/die Autor(en) 2018. Dieser Artikel ist eine Open-Access-Publikation.

Zusammenfassung Eine meist divergente Augenstellung von Skulpturen ist vom Künstler beabsichtigt und keine Fehlleistung. Im gesamten Zeitraum von 2500 a. Chr. bis 1800 p. Chr. ist sie ein Zeichen von Krankheit, Tod oder irrealen Geistwesen. Ab 1300 überwiegt in Europa die christliche Spiritualität: Viele Heiligengestalten scheinen geistig in ein besseres Jenseits entrückt, erkennbar an ihren nach außen oben gerichteten Augen. Bei Figurengruppen dient verschiedene Blickrichtung beider Augen zur Kontaktaufnahme untereinander. In der Spätgotik (um 1500 p. Chr.), beginnend mit V. Stoß, und im Barock drückt die Augenstellung Emotionen aller Arten aus. Aus alten medizinischen Büchern wissen wir, dass den einzelnen Augenmuskeln bestimmte emotionale Funktionen zugeordnet wurden. Mit der Aufklärung bricht auch in der Kunst eine neue Zeit an.

Schlüsselwörter Divergenz-Schielen und Kunst . Skulpturen als Ausdrucksmittel - Todes-Darstellung · Emotionen, fixiert in Stein oder Holz - Stilperioden der Kunst

\section{The statements made by anomalous eye positions in European fine art}

Summary Divergent eye positions in sculptures are not errors, but intended by the artist. These squinting eyes indicated the presence of illness, death, or unreal persons and ghosts during the centuries be-

\footnotetext{
Nach einem Vortrag vor dem Ärztekunstverein Österreichs, Mai 2013. Die Zahl der Abbildungen wurde für den Druck wesentlich reduziert.

ao. Univ.-Prof. Dr. E. Stangler-Zuschrott ( $ه)$

Augenheilkunde und Optometrie, Univ.-Augenklinik Wien,

Landstrasser Hauptstraße 141/11, Wien, Österreich

e.stangler-zuschrott@aon.at
}

tween $2500 \mathrm{BC}$ and $1800 \mathrm{AD}$. After $1300 \mathrm{AD}$, the spirit of Christianity spread over Europe; many statues of saints were created, their eyes typically looking upwards and outwards as a sign of transcendence into a better world. In groups of figures we sometimes find sculptures turning each eye in a different direction to make contact with other persons. In the late gothic period (around $1500 \mathrm{AD}$ ) and the Baroque era, the artists began to express all sorts of emotions by different eye positions, above all Veit Stoß in his scenic reliefs. We know from ancient medical books that specific emotional functions were ascribed to individual eye muscles. The Age of Enlightenment changed everything, even the fine arts.

Keywords Divergent squint and fine arts - Sculptures, expressive - Death, represented - Emotions, fixed in stone or wood $\cdot$ Style periods of fine arts

\section{Problemstellung}

Ein strabologisch geschulter Blick ist selbst im Urlaub prüfend auf Augenpaare von lebenden Personen wie auch von Statuen gerichtet. Das Schlüsselerlebnis $\mathrm{zu}$ dieser Studie fand vor etlichen Jahren in der Pfarrkirche von Virgen/Osttirol statt. Zwei kunstvolle Statuen [1] fesseln die Aufmerksamkeit der Besucher: Sankt Katharina schaut mit seligem Gesichtsausdruck und leicht divergenten Augen nach oben, als sähe sie den Himmel offen. Ihre Kollegin Barbara hingegen hat die Augen stark divergent nach unten gerichtet (Abb. 1), warum wohl? Die diagnostischen Regeln der Strabismologie gelten nicht für Statuen; ein unabsichtlicher Fehler des Künstlers ist ebenfalls auszuschließen. Sankt Barbara bleibt zunächst ein Rätsel. Vom Thema gefesselt, begann ein ständiges Suchen mit der Kamera nach einschlägigen Objekten in Kirchen, Klöstern, Museen und ein Blättern in Büchern. 


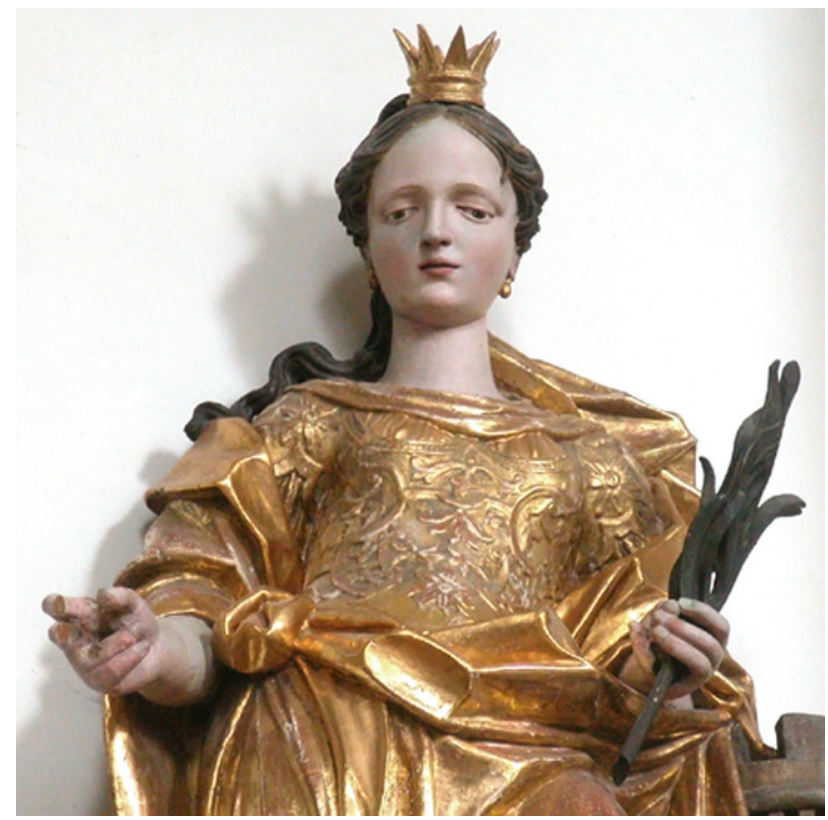

Abb. 1 HI. Barbara, Pfarrkirche Virgen, Osttirol, J. Paterer 1750. (Mit freundl. Genehmigung der Zeitschrift ophta/u. novotny fachverlag, Bottighofen am Bodensee, Schweiz)

\section{Methodik}

Die Augenstellung von Statuen zu beurteilen ist schwieriger als bei Gemälden, bei denen oft Lichtpunkte als Markierung verwendet werden. Eine Fotografie sollte möglichst frontal erfolgen, um parallaktische Fehleinschätzungen zu vermeiden. Einen Strabismus zu diagnostizieren, gelingt besser bei polychromierten als bei ungefassten Figuren, bei denen Limbus und Pupille, im Holz graviert, den Blick festlegen; am häufigsten findet man Plastiken aus reinem Lindenholz bei Tilman Riemenschneider, der zwar Holzschnitzer, aber kein gewerblicher „Fassmaler“ war. Zu jener Zeit galten Künstler als Gewerbetreibende.

Es folgt die Vorstellung von Einzelobjekten aus unterschiedlichen Zeitperioden zwischen 2500 a. Chr. und 1800 p. Chr., aus dem europäischen und benachbarten Kulturraum.

\section{Die Antike}

Aus den hochentwickelten Kulturen Vorderasiens ist mir kein Beispiel einer schielenden Skulptur bekannt, doch sei eine besonders eindrucksvolle Statue erwähnt, der Mann aus Mari, (Louvre, um 2200 a. Chr.). Seine Augen sind in korrekter Stellung aus Bitumen eingelegt, die Lidränder geschwärzt, wie es auch heute die Mode ist, das Gewand stilisiert. Das Besondere ist sein feines, zeitloses Lächeln, womit er den Betrachter über Jahrtausende hinweg anblickt und die Ereignisse der Gegenwart relativiert und distanziert.

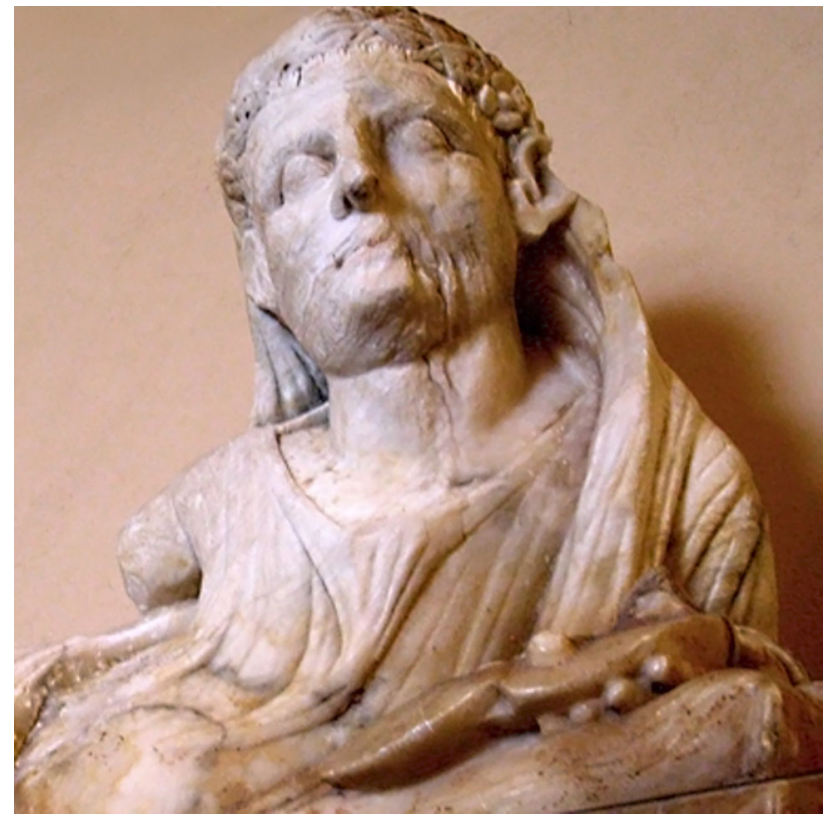

Abb. 2 Der Wahrsager Aule Lecu, Museum Guarnacci in Volterra. 2.-1. Jhdt a. Chr. (Mit freundl. Genehmigung der Zeitschrift ophta/u. novotny fachverlag, Bottighofen am Bodensee, Schweiz)

In Ägypten herrschten strenge Vorschriften betreffend die Fertigung von Skulpturen, die stets die herrschende Oberschicht darstellten. Eine Schielstellung der Augen findet man bei ihnen nicht. Dennoch gibt es divergent Schielende, es sind dies irreale Gestalten, Grabbeigaben, die erst im jenseitigen Leben ihrem Herrn dienen sollten: Uschebtis, Matrosen auf einem Segelschiff, Mädchen in der Spinnstube usw.

Aus dem Klassischen Zeitalter Griechenlands ist der Autorin keine Skulptur mit schielenden Augen bekannt.

Etwa zur gleichen Zeit befand sich auch Etrurien im „goldenen Zeitalter“. Ein Beispiel für einen glücklichen Gesichtsausdruck: Der Hermes von Veji (um 500 a. Chr.) hat parallele blaue Augen und ein Lächeln ähnlich jenem des Mannes aus Mari [2]. In den folgenden Jahrhunderten gerieten die etruskischen Stadtstaaten zunehmend durch die Römer in Bedrängnis, erkenntlich an den besorgten Gesichtern der Skulpturen auf den Sarkophagen im Museum Guarnacci, Volterra. Die Etrusker wollten ihre eigene Person möglichst lebensecht der Nachwelt überliefern, kein auf Sarkophagen Dargestellter schielt, mit wenigen Ausnahmen: Eine leichte Divergenz findet man bei einem verstorbenen Kind und einem offenbar kranken Mann, der auf dem Sarkophag nicht wie üblich den Oberkörper aufrecht hält, sondern den Kopf auf einem Polster lagert. Der Wahrsager Aule Lecu ist verschleiert, seine Augen in Trance nach außen abgewichen, er hält eine Schafsleber in der Hand (Abb. 2.). Im Museum von Volterra befindet sich die Totenstele einer Frau mit divergenten Augen und über der Brust nach oben gekreuzten Armen, einer Trauergeste. 
Die Etrusker glaubten an ein Fortleben nach dem Tod. Um der Seele eine schützende Wohnung auf ihrer gefährlichen Reise ins Jenseits zu bieten, fertigten sie Urnen mit menschenähnlichen Köpfen an. Meist sind deren Augen parallel, denn die Seele ist nicht tot, sie befindet sich auf Wanderung. Divergenz scheint also mit dem Tod des Körpers verbunden zu sein und ist auch bei jenen Geistern vorhanden, die den Toten bewachen sollten [3]; es handelt sich um eine Skulptur aus Bronze aus den Vatikanischen Museen, (7. Jhdt a. Chr.).

Die Kunst des Römischen Reiches ist an jener der Griechen orientiert, sie bietet keine Funde für diese Studie.

\section{Die Romanik}

Charakteristisch für diese Zeit ist die Darstellung der Pupille als „Sehloch“. Schielende findet man nur ausnahmsweise. Ein Beispiel bietet die Pfarrkirche von Schöngrabern/NÖ (Niederösterreich) in der Versu-

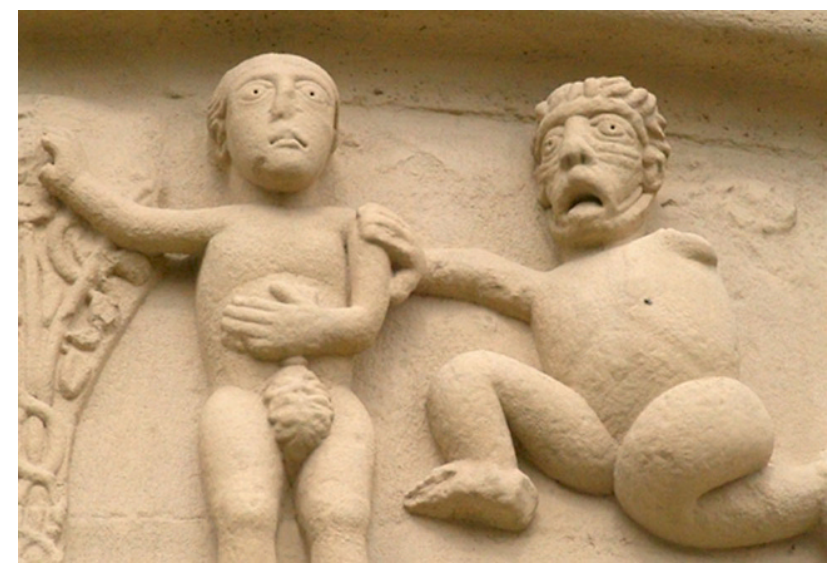

Abb. 3 Der Sündenfall. Relief an der Außenseite der Pfarrkirche Schöngrabern, NÖ, 1210-1230 (Mit freundl. Genehmigung der Zeitschrift ophta/u. novotny fachverlag, Bottighofen am Bodensee, Schweiz)

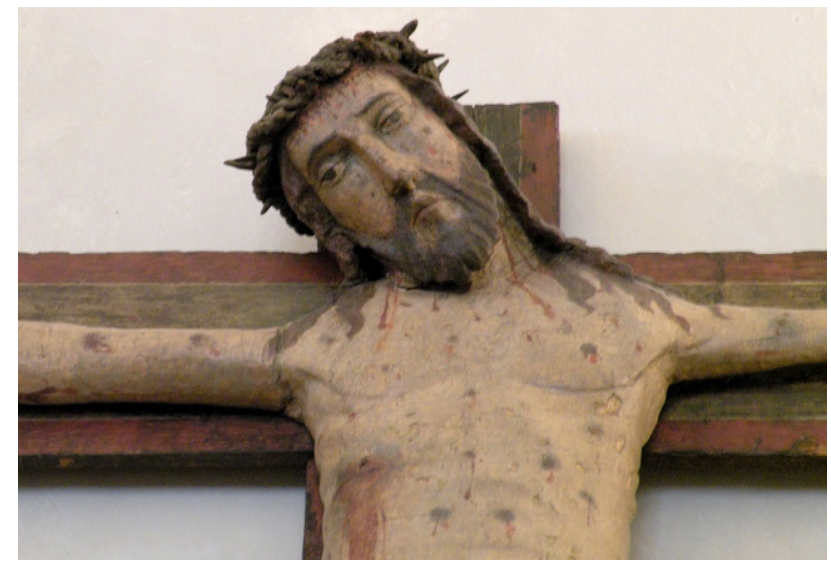

Abb. 4 Kruzifix, Oberfranken, 1240-1250, Bayrisches Nationalmuseum München (@ E. Stangler-Zuschrott)

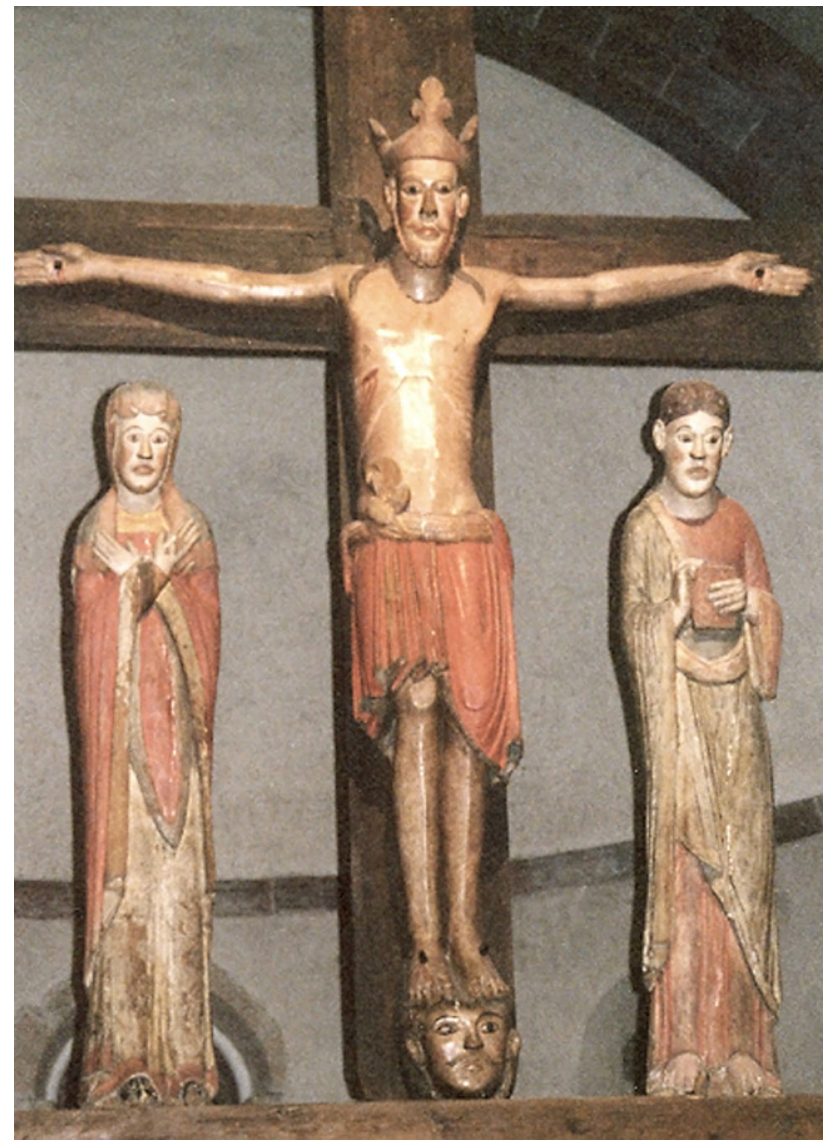

Abb. 5 Kreuzigungsgruppe im Dom von Innichen/Südtirol, um 1200 (○ E. Stangler-Zuschrott)

chung Adams (nicht Evas!): Der einem Tier ähnliche Teufel schielt divergent, nicht der Mensch (Abb. 3).

Als höchstes Heiligtum wird im Dom zu Lucca seit dem 8. Jhdt. der „Volto Santo“ verehrt, eine Christusfigur am Kreuz mit extrem divergenten und höhendifferenten Augen. Die Füße, wie in der Romanik üblich, auf einem Podest stehend. In vielen Museen und obligat in allen christlichen Kirchen befinden sich Darstellungen von Christus am Kreuz. Ohne Bezug auf die Entstehungszeit zeigt dieser entweder geschlossene Augen oder aber, nicht selten, divergent stehende als Zeichen des Todeskampfes (Abb. 4). Ein anderes spirituelles Konzept liegt den romanischen Triumphkreuzen zugrunde, wie z. B. der Kreuzigungsgruppe von Innichen (Abb. 5). Der gekrönte Christus am Kreuz ist der Herr des Lebens und Sieger über den Tod; er hat weit offene, parallele Augen, ebenso die Begleitfiguren unter dem Kreuz. Eine ähnliche Christusdarstellung findet man u. a. in der Abbadia di San Salvatore/ Siena.

Romanische Madonnen sitzen in einem Thronsessel mit ihrem Kind am Schoß, das sie dem Betrachter als Mittelpunkt der gesamten Darstellung präsentieren, auch im spirituellen Sinn (Abb. 6). Sie alle zeigen parallele Augen. 


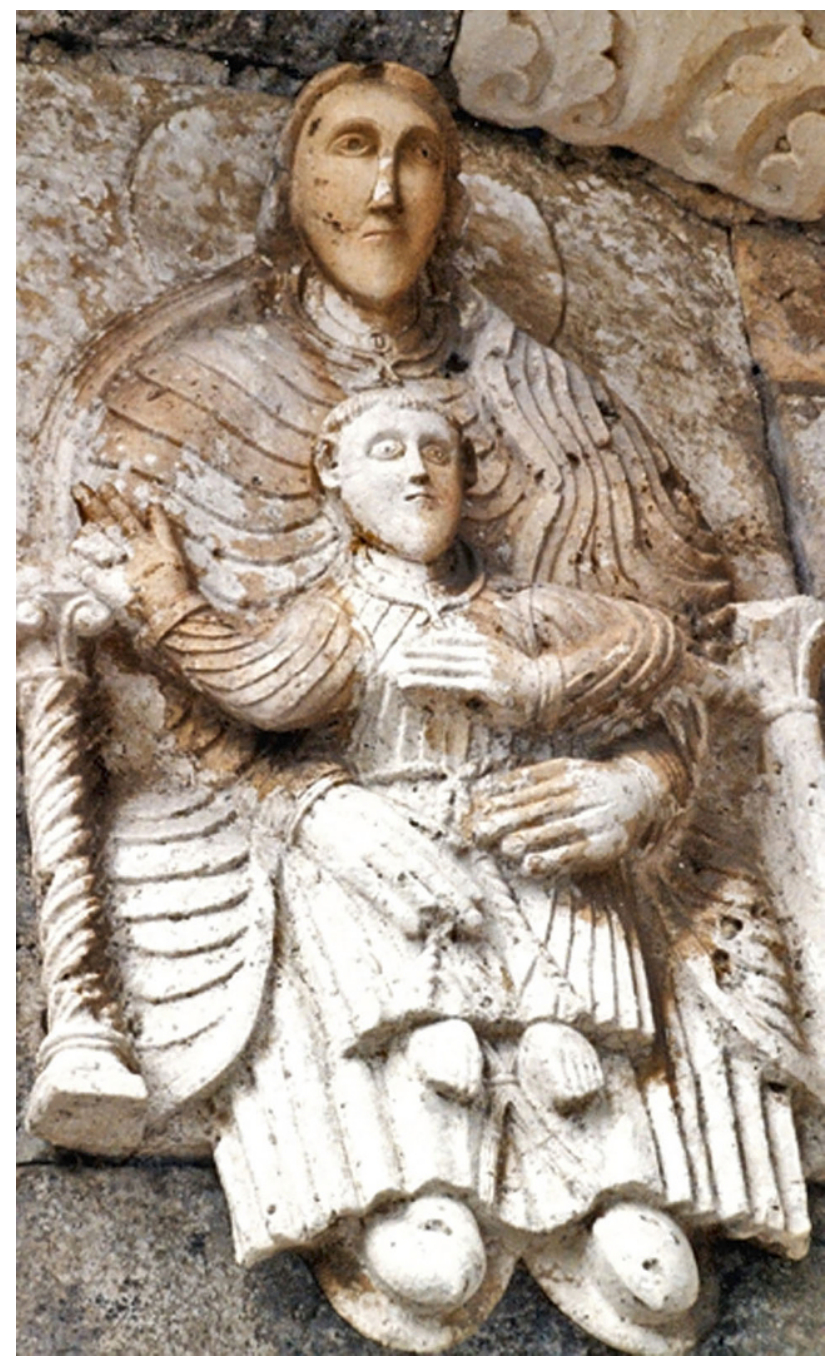

Abb. 6 Madonna mit Kind, Portal der Kirche Santa Maria Maggiore von Tuscania/Italien, um 1200 (@ E. StanglerZuschrott)

\section{Die Gotik}

In diesem Zeitalter findet man am häufigsten Skulpturen mit divergenter Augenstellung, deren Bedeutung unterschiedlich ist. Konvergent schielende Figuren sind seltene Ausnahmen.

\section{Die Augenstellung zur Kontaktaufnahme oder als Hinweis}

In der Gotik ändert sich die Darstellung einer Mutter Gottes mit Kind gegenüber der Romanik, das Kind wird auf die Seite gerückt in den Arm der Mutter. Diese schaut manchmal ihr Kind an, meist aber zum gläubigen Publikum. Nicht selten findet man eine Zwischenlösung: Ein Auge blickt geradeaus, das andere auf das Kind, woraus sich ein Schielstellung ergibt (Abb. 7). Je eine Bezugsperson links und rechts führt zu extremer Abweichung der Augen einer Statue. Dabei ist nach Texten der Bibel anzunehmen, dass Begegnungen mit einem Dialog verbunden sind. Ein berühmtes Beispiel

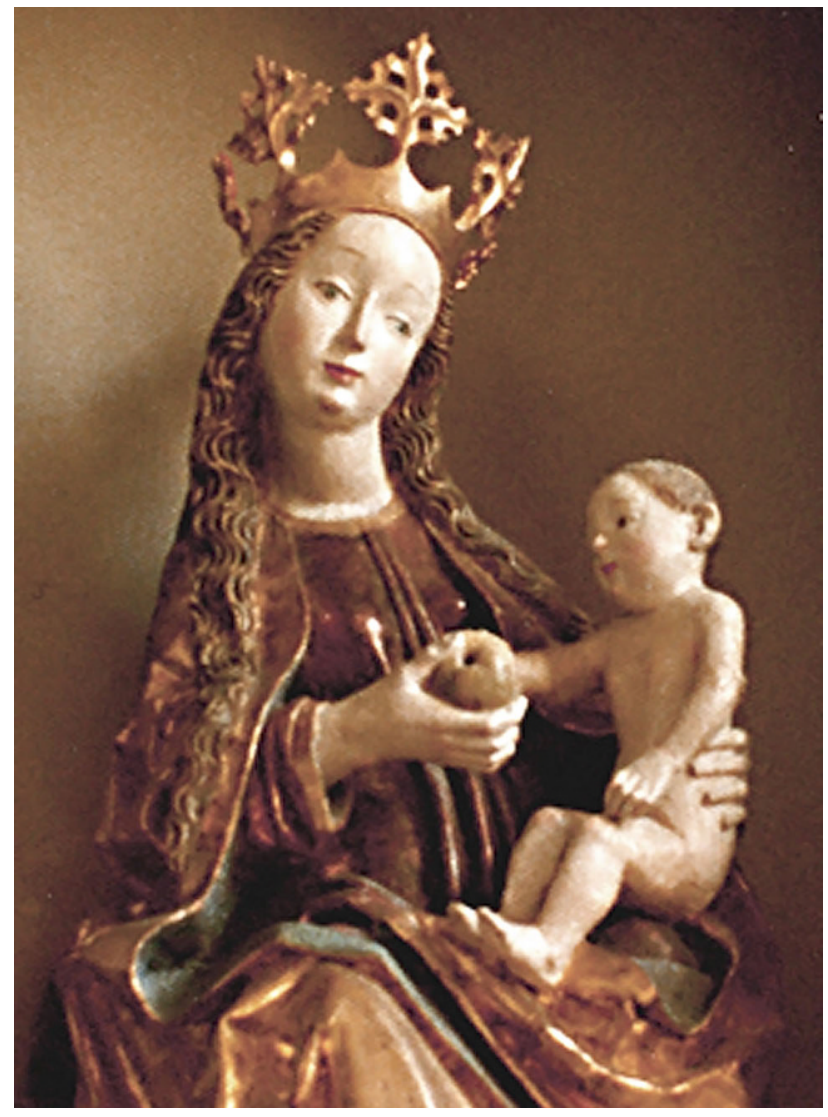

Abb. 7 Madonna von Prigglitz/NÖ, um 1400. Dom-Museum, Wien (@ E. Stangler-Zuschrott)

ist der „Englische Gruß“ des Veit Stoß in Nürnberg, St. Lorenzkirche: Der Verkündigungsengel begrüßt Maria, bei seitlicher Anordnung sehen beide einander mit jeweils einem divergenten Auge an.

\section{Divergenzstellung als Zeichen der Verklärung und} Entrückung in das Jenseits

Engel als Geistwesen haben besonders in den Werken Riemenschneiders stets divergente Augen. Man findet sie auch in St. Stephan/Wien (Abb. 8). Isoliert stehende "schielende“ Heiligenfiguren befinden sich geistig in einer anderen Welt. Die Reliquienbüste der Hl. Afra hat 2 Gründe divergent zu schauen: Tod und Verklärung (Abb. 9). Ein besonderes Beispiel bieten die Reliefs des Marienaltars in Creglingen von T. Riemenschneider: Während ihres Erdenlebens hat Maria parallele Augen, sobald sie aber zum Himmel erhoben wird, zeigt sie nur noch Divergenz.

\section{Divergenzstellung als Ausdruck von Emotion, in Gotik und Barockzeitalter}

Schweres seelisches Leid zeigen jetzt die unter dem Kreuz stehenden Personen, Maria und Johannes bei vielen Kreuzigungsgruppen. Im Passionsaltar von Riemenschneider schaut sogar ein römischer Soldat mit einem divergenten Auge nach oben auf den Gekreuzigten, wohl nicht aus Trauer, eher aus Angst; nach 


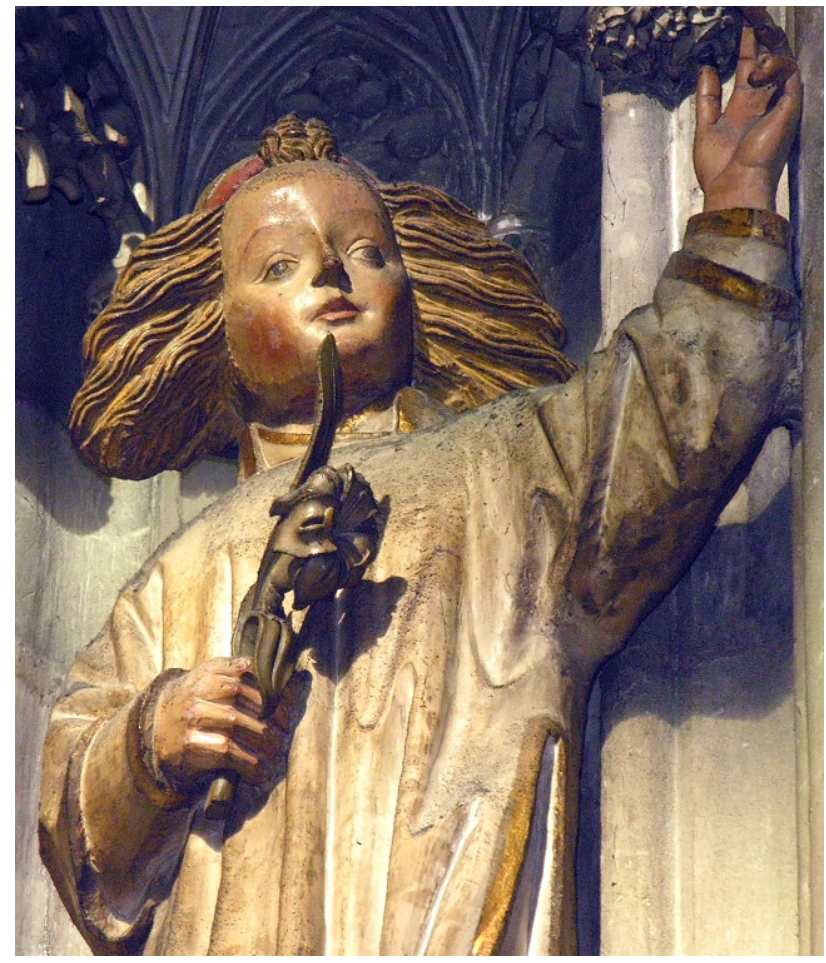

Abb. 8 Verkündigungsengel, Stephansdom Wien, um 1450 (C E. Stangler-Zuschrott)

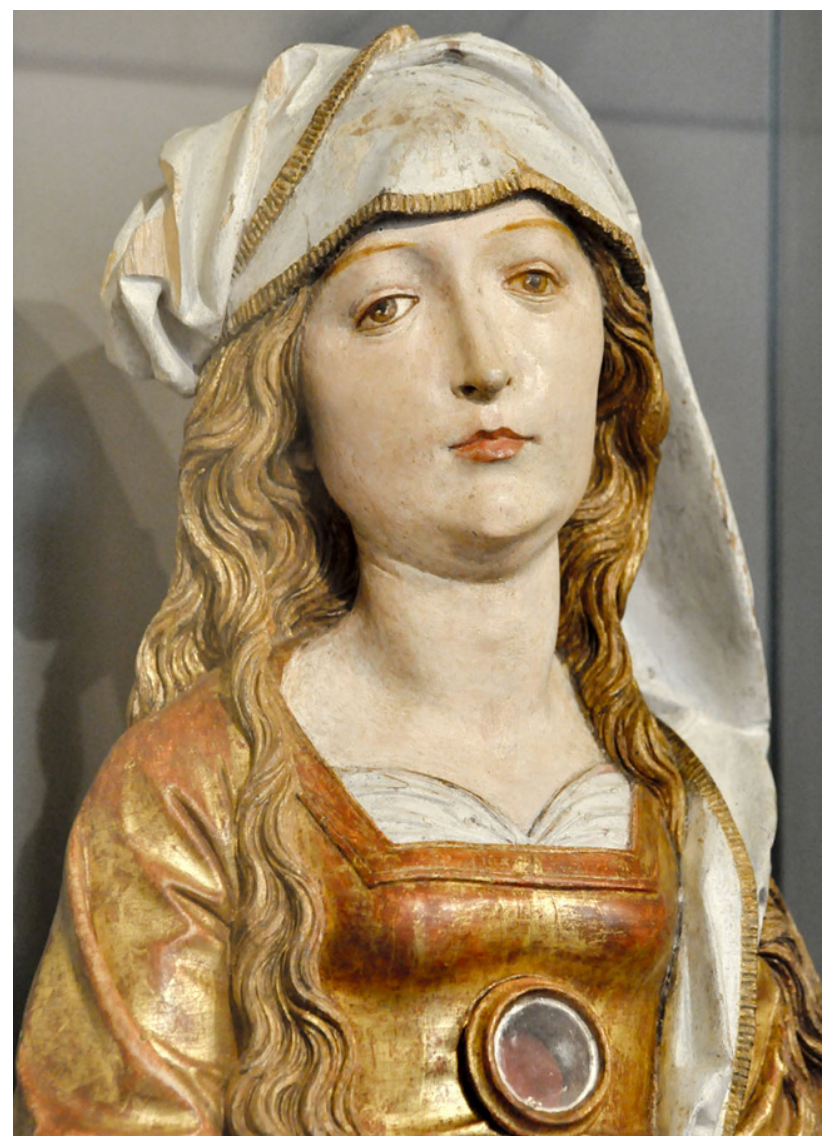

Abb. 9 HI. Afra, Reliquienbüste, Tilman Riemenschneider, um 1499. Bayrisches Nationalmuseum München (๑ E. StanglerZuschrott)

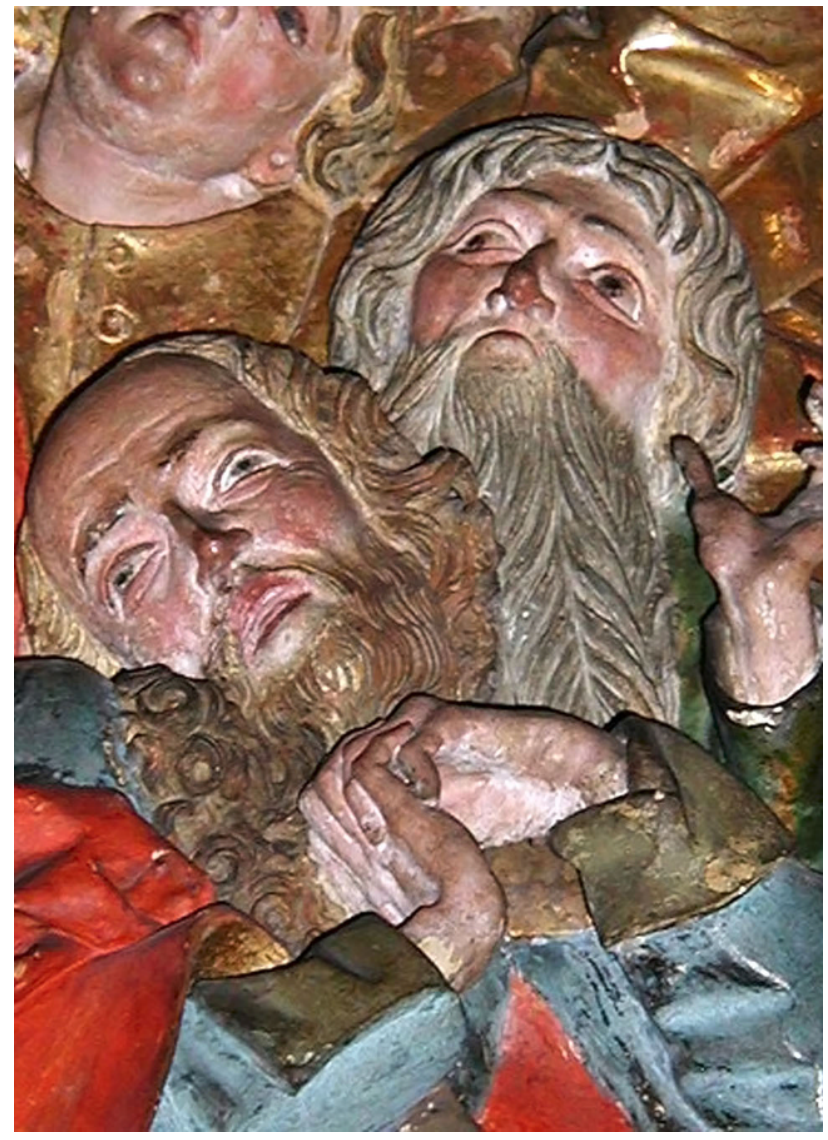

Abb. 10 Detail aus dem „Marientod“ des Salzburger Marienretabels von Veit Stoß, 1498, im Stift Nonnberg, Salzburg (@ E. Stangler-Zuschrott)

Angaben der Bibel hatte er erkannt, Gottes Sohn hingerichtet zu haben, und muss nun die Rache des Gottes (Zeus oder der Gott der Juden?) fürchten. Anders ist die Situation bei Vesperbildern. Unter sehr vielen besichtigten Plastiken von Maria mit ihrem toten Sohn im Schoß wurde bisher keine Mutter Gottes, sondern fallweise nur der tote Jesus mit divergenten Augen gefunden. Eine psychologische Erklärung wäre denkbar: Das akute dramatische Geschehen ist vorbei, das übergroße Leid macht die Seele empfindungslos, die Tränen versiegen.

Entsetzen und Verzweiflung spiegelt sich in den $\mathrm{Au}-$ gen der Apostel wider, die Maria bei ihrem Ableben händeringend umgeben. Der Marientod war ein beliebtes Thema für Reliefs in der Spätgotik (Abb. 10).

Die Tugend der Demut äußert sich im Blick nach unten und außen. Die klassischen Beispiele dafür sind etliche spätgotische Schnitzaltäre, welche die Krönung Mariens im Himmel darstellen (Abb. 11).

Körperliche Schmerzen und Todesqualen verändern die Augenstellung in Richtung Divergenz. Beliebt ist die Darstellung des römischen Soldaten Sebastian (Abb. 12), der jedoch nicht immer zu leiden scheint; manchmal hat man den Eindruck, er freue sich auf das Paradies; dann aber schielt er auch nicht. Sehr 


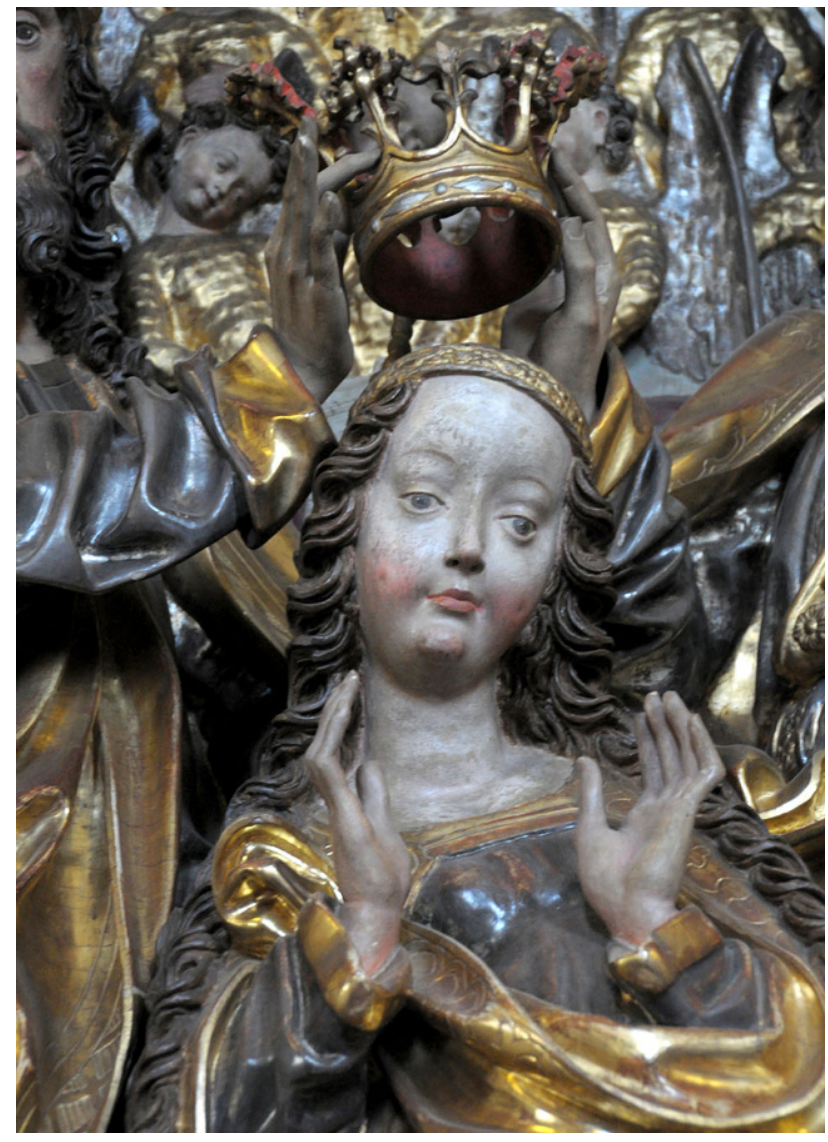

Abb. 11 Krönung Mariens. Detail aus dem Flügelaltar von Maria Gail bei Villach. 1514-1515, Villacher Werkstätte (@ E. Stangler-Zuschrott)

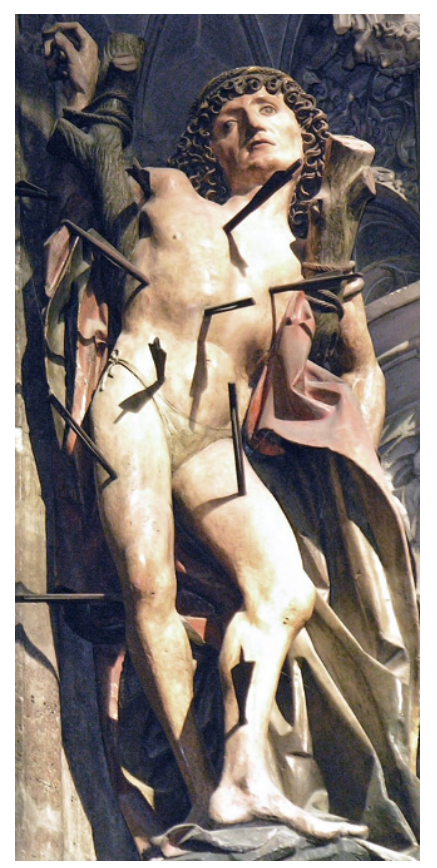

Abb. 12 St. Sebastian, Stephansdom Wien, um 1450 (C E. Stangler-Zuschrott)

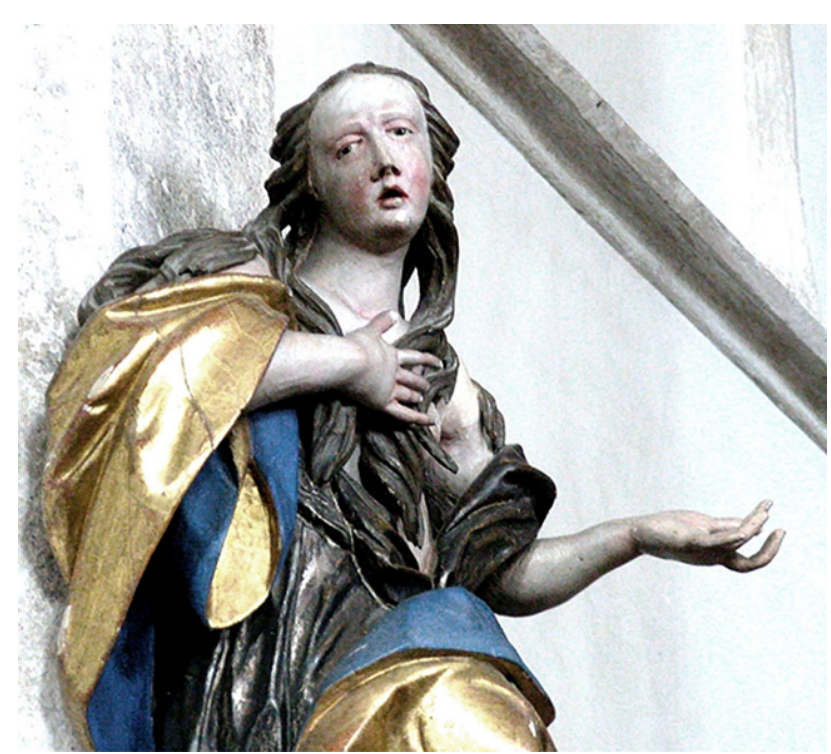

Abb. 13 Maria Magdalena, Patronin der Sünder, 18. Jhdt., Pfarrkirche Schönbach/NÖ (@ E. Stangler-Zuschrott)

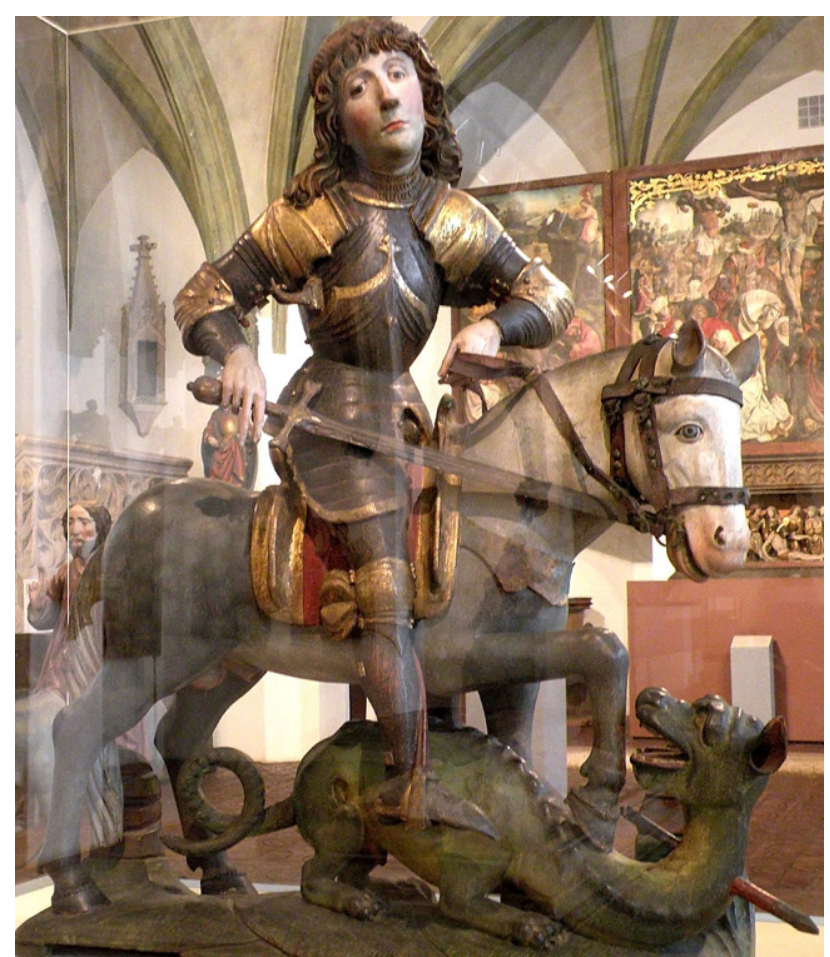

Abb. 14 St. Georg zu Pferd, Brixen, um 1500, Bayrisches Nationalmuseum München (๑ E. Stangler-Zuschrott)

eindrucksvoll ist St. Veit im Ölkessel von V. Stoß im Germanischen Nationalmuseum Nürnberg.

Reue kann ebenfalls Divergenz hervorrufen. Vereinzelt findet man auf Beichtstühlen das Abbild einer Sünderin, die sich auf die Brust klopft (Abb. 13). Die hübsche törichte Jungfrau des Schnatterpeck-Altars in Niederlana (Südtirol) weint, weil sie die Ankunft des Bräutigams versäumt hat. 


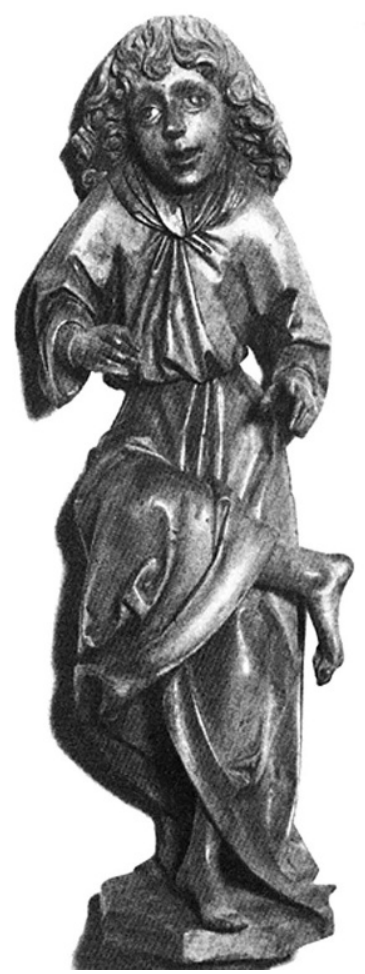

Abb. 15 Tanzende Figur, Detail aus dem Flügelaltar von Kefermarkt/OÖ, um 1497 (C E. Stangler-Zuschrott)

Heftige Gemütserregung und Stress zeigt sich als Divergenz der Augen. Sankt Georg, der Drachentöter hat allen Grund zu schielen (Abb. 14). Glücklicherweise gibt es auch positive Stimmungen, die sich in den Augen widerspiegeln, wie z. B. Lebensfreunde bei fröhlichem Musizieren und Tanzen (Abb. 15).

\section{Divergenzstellung als Botschaft}

Wie ist nun, aufgrund der bisherigen Einsichten, die Augenstellung der barocken St. Barbara aus Virgen einzuschätzen (Abb. 1)? Betrachtet man die Figur genau, sieht man, dass an der rechten Hand 2 Finger abgebrochen sind; vermutlich hielt sie früher darin ein Schwert als Kennzeichen ihres gewaltsamen Todes. Ihr rechtes Auge ist auf diese Hand gerichtet, das linke auf die linke Hand, in der sie die Siegespalme hält. Der gedankliche Zusammenhang scheint durch die Blickrichtung als Aussage erkennbar: Durch Leiden und Tod gelangt man zur ewigen Seligkeit.

\section{Darstellung historischer Personen mit vermutlich tatsächlichem Schielfehler}

Der Dombaumeister Anton Pilgram von St. Stephan/ Wien war auch Steinmetz und hat sich im Dom selbst porträtiert (1513). Sein linkes Auge steht deutlich divergent, unabhängig davon, aus welcher Richtung fotografiert wird. Historisch ist darüber nichts bekannt, die Vermutung liegt jedoch nahe, dass eine perforierende Verletzung mit traumatischer Katarakt das Auge nach außen abdriften ließ. Im Gegensatz dazu fällt bei

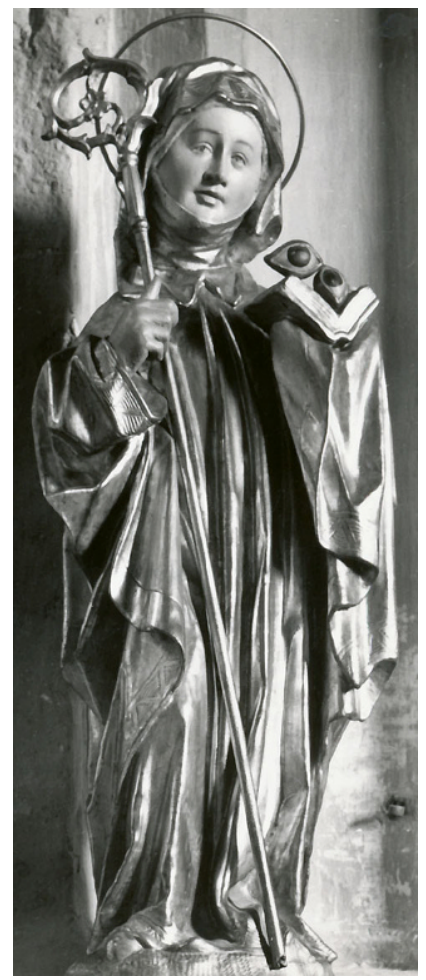

Abb. 16 HI. Odilia aus dem Stift Nonnberg, Herkunft unbekannt, um 1500 (Postkarte von 1963) (C E. Stangler-Zuschrott)

St. Wolfgang des Altars von Michael Pacher bei seitlicher Anordnung der Figur eine Schielstellung perspektivisch nicht auf, nur bei frontaler Sicht.

Eine interessante Persönlichkeit ist die als Odilia bzw. Ottilie bekannte Heilige, die bei Augenerkrankungen angerufen wird. Der Legende nach war sie blind geboren, vom adeligen Vater verstoßen, in ein Kloster aufgenommen und durch die Taufe sehend geworden. Später war sie selbst Äbtissin eines Klosters im Elsass, einem bekannten Wallfahrtsort. 1963 erhielt ich eine Ansichtskarte aus Salzburg mit dem Bild einer Odilienstatue, die sich im Kloster Nonnberg in Salzburg befindet (Abb. 16). Die Heilige mit dem Stab einer Äbtissin zeigt die Zeichen eines kongenitalen Schielsyndroms: Strabismus convergens et sursumvergens links und Kopfneigung zur linken Schulter; als spezielles Symbol hält sie ein Buch mit einem Augenpaar, das analoge Schielstellung zeigt. Leider sind die Augen dieser Statue im Zuge einer Restaurierung „gerade" gerichtet worden. In der Pfarrkirche von Amlach bei Lienz/Osttirol befindet sich eine Odilien-Statue mit ebenfalls leicht konvergentem linkem Auge; ein Zufall oder Historie?

\section{Schlussfolgerungen}

Eine irreguläre Augenstellung plastischer Kunstwerke wird i. Allg. vom Publikum nicht wahrgenommen.

Aus der Literatur erfährt man fast nichts über dieses Thema, einzig Trevor-Roper [4] meint, eine Augenabweichung sei nicht immer realistisch, schwaches Aus- 


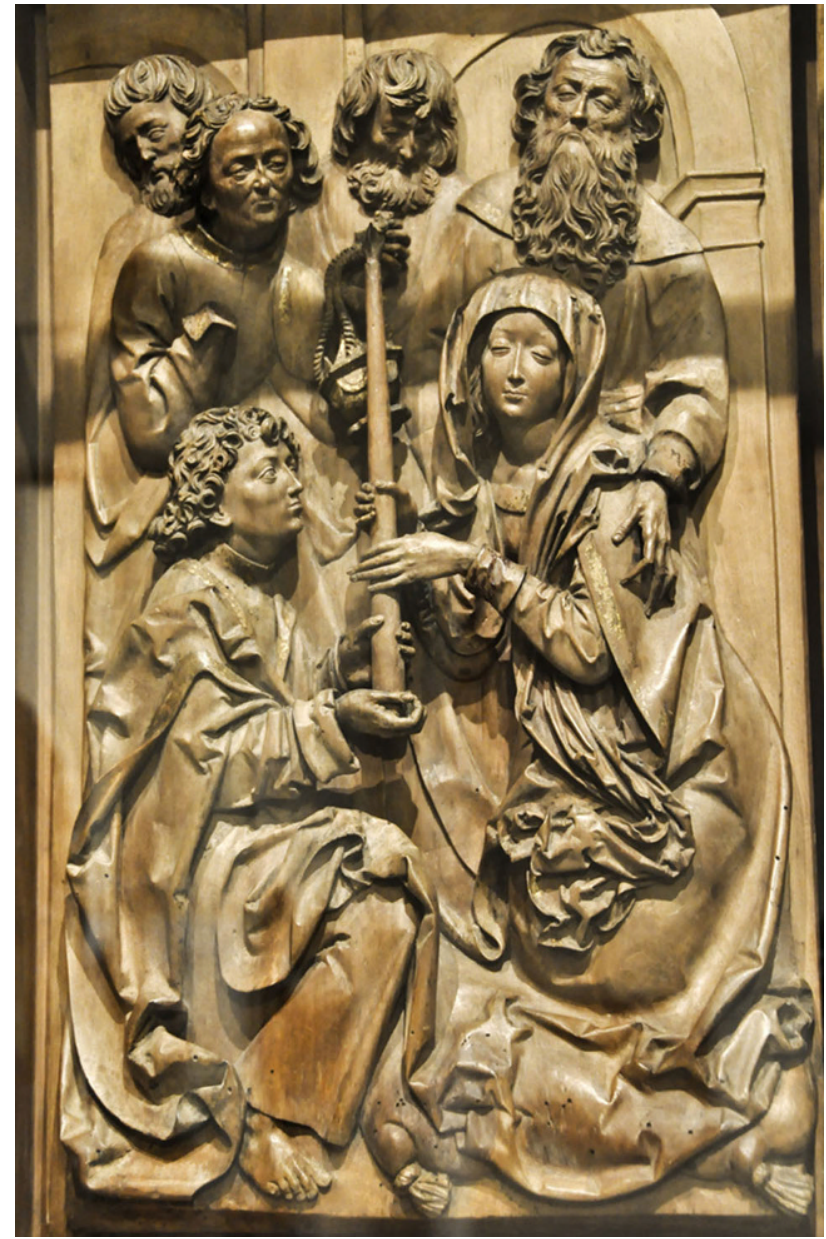

Abb. 17 Marientod, letztes Werk des Veit Stoß, 1523. Bayrisches Nationalmuseum München (ㄷ E. Stangler-Zuschrott)

wärtsschielen vermittle einen vergeistigten Ausdruck. Kollege Mühlendyk [5] hat sich ebenfalls mit dem Thema befasst. Eigene frühere Publikationen [6, 7] werden hier zusammengefasst und mit neuem Bildmaterial ergänzt.

In der vorliegenden Studie zieht sich folgende Erkenntnis wie ein roter Faden von der Antike bis zum Ende des Barock: Divergenzstellung der Augen ist ein Attribut von Tod und Krankheit, von irrealen Personen und Geistwesen. Tatsächlich ist die Augenstellung Toter leicht divergent [8].

Mit der Verbreitung des Christentums in Europa unterlagen die Künstler früherer Jahrhunderte spirituellen Einflüssen, der Großteil aller relevanten Objekte stammt aus Kirchen und Klöstern, zu datieren zwischen 1300 und 1800. Vor der Erfindung des Buchdrucks und in Zeiten mangelnder Schulbildung waren plastische Darstellungen, ähnlich den Fastentüchern jener Zeit, wohl als Biblia pauperum gedacht.

In Übereinstimmung mit Trevor-Roper finden wir bei isoliert stehenden Heiligenstatuen divergente Augen als Zeichen von Entrückung und Ekstase. Anders bei Gruppenplastiken oder Darstellungen einer Szene. Gotische Statuen zeigen oft beide Augen in unter- schiedlicher Blickrichtung. Dies zur Kontaktaufnahme mit anderen Personen, zum Dialog oder als Hinweis auf eine zweite Person. Schauen beide Augen unterschiedliche Gegenstände an, ist ein gedanklicher $\mathrm{Zu}$ sammenhang zwischen beiden Objekten zu erwägen.

Erst zur Zeit von Spätgotik und Barock wirken plastische Darstellungen zunehmend lebendiger, die Figuren erscheinen bewegt, und v. a. zeigen sie Emotionen, welche nicht nur aus dem Inhalt der Szene, sondern auch an der Augenstellung zu erkennen sind.

Eine Bestätigung dieser Auffassung findet sich in der alten medizinischen Literatur bei Valentin Kräutermann, 1732 [9]. Daraus sind einige Originalzitate entnommen:

Sechs Musculi oder Mäusslein, so zur Bewegung der Augen dienen, zehlen die Anatomici, als vier gerade und zwey krumme, deren Nahmen von ihrem Lager und Gebrauch genommen sind. Der Erste, Attollens oder Superbus, aliis Pharisaicus, der Stolze genannt, zeucht das Auge hinaufwerts. Der Andere Gerade, lieget unterhalb dem Auge, dem vorigen gantz entgegen, heist Deprimens, Publicanus, humilis, der Demüthige, dieser lencket das Auge herunterwerts. Der Dritte führet das Auge einwerts (lat. Musculus Adducens, Bibitorius) nach der Nase zu, wird auch das Lese-Mäusslein genannt. Der Vierdte führet das Auge auswerts, Abducens oder Indignatorius, das abführende oder verachtende Mäusslein genennt. Der Fünfte und Sechste werden Obliqui, Trochleares, Krumme Mäusslein, auch die Verliebten oder Liebes-Musculi geheissen. [Beschreibung der 4. Hirnnerven]: Den Namen Pathetici Nervi oder Leidenden hat man (ihm) zugeeignet, dieweil man glaubet, dass es diejenigen Wendungen der Augen, so sich in der Liebe, in dem Hass, in Furcht, Zorn, Traurigkeit und anderen Gemüths Passionen äussern, wider Willen erreget; zum Unterschiede des Augen-bewegenden [3. Nervenpaares], welches nur seine Dienste leistet, wenn man eine Ursach mit Ernst anschauen will.

Die Persönlichkeit des Künstlers ist nicht zuletzt entscheidend für die Art der Darstellung. Ein Vergleich zweier Zeitgenossen der Spätgotik ist aufschlussreich, die in ihrem Werk wesentliche Unterschiede aufweisen, sowohl in der Gestaltung als auch in der Aussage durch die Augenstellung. In Riemenschneiders Arbeiten findet man eher selten den Ausdruck von Emotionen oder von Blickkontakten. Sein Leben war von der Religion geprägt, sein Werk erinnert die Menschen an ihr Seelenheil. Seine Heiligenfiguren, Apostel und Engel zeigen divergente Augen als Zeichen der Transzendenz in ein überirdisches Sein. Veit Stoß hingegen ist ein Dramatiker. Er hat die meisten Schielenden geschaffen, bei ihm dominieren Bewegung und Emotion: Flatternde Gewänder, verzweifelte Apostel beim Tod Mariens und tiefstes Leid unter dem Kreuz Christi. Fast erblindet schuf Stoß sein letztes Werk, einen 
Marientod; darin haben fast alle Personen die Augen geschlossen. - Licht und Leben erlöschen im Künstler, seine Schnitzkunst ist immer noch perfekt (Abb. 17).

Danksagung Herrn Ing. Reinhard Müller danke ich herzlich für die technische Bearbeitung des Manuskripts.

Interessenkonflikt E. Stangler-Zuschrott gibt an, dass kein Interessenkonflikt besteht.

Open Access This article is distributed under the terms of the Creative Commons Attribution 4.0 International License (http://creativecommons.org/licenses/by/4.0/), which permits unrestricted use, distribution, and reproduction in any medium, provided you give appropriate credit to the original author(s) and the source, provide a link to the Creative Commons license, and indicate if changes were made.

\section{Literatur}

1. Bundesdenkmalamt Wien. DEHIO-Handbuch der KunstdenkmälerÖsterreichs, Tirol. Wien:Anton Schroll; 1980.

2. Cristofani M. Die Etrusker. Geheimnisvolle Kultur im antiken Italien. Stuttgart: Belser; 2006.
3. Hamblin J. Die Etrusker. Die Frühzeit des Menschen. New York: Time-lifeinternational; 1977.

4. Trevor-Roper P, Redwald H. Der veränderte Blick. Über den EinflussvonSehfehlern auf Kunstund Charakter. Hamburg: dtv;2001.

5. Mühlendyk H. Schielen in der christlichen Kunst. Z Prakt Augenheilkd.2004;25:315-20.

6. Stangler-Zuschrott E. Augenstellung, Augendarstellung und Blickrichtung in der Bildenden Kunst. Ophta. 2005;(5):9-17.

7. Stangler-Zuschrott E. Die Augenstellung und ihre Bedeutung in den Meisterwerken spätgotischer Schnitzkunst. ZPraktAugenheilkd.2010;31:411-8.

8. Kaufmann H. Strabismus. 3. Aufl. Stuttgart:Thieme; 2004.

9. KräutermannV.DersichereAugen-undZahnarzt, Arnstadt. Berlin: Nationales Druckhaus; 1983. S. 3-5. Erstausgabe 1732.

ao. Univ.-Prof. Dr. Elfriede Stangler-Zuschrott, ao. Univ.Prof. Dr. E. Stangler-Zuschrott ist nicht mehr aktiv an der Augenklinik tätig, aber publizistisch weiterhin mit der Augenheilkundeverbunden. 